\title{
Homoepitaxial nonpolar (10-10) ZnO/ZnMgO monolithic microcavities: Towards reduced photonic disorder
}

J. Zuniga-Perez, L. Kappei, C. Deparis, F. Reveret, M. Grundmann, E. de Prado, O. Jamadi, J. Leymarie, S. Chenot, and M. Leroux

Citation: Appl. Phys. Lett. 108, 251904 (2016); doi: 10.1063/1.4954796

View online: https://doi.org/10.1063/1.4954796

View Table of Contents: http://aip.scitation.org/toc/apl/108/25

Published by the American Institute of Physics

\section{Articles you may be interested in}

Polarity in GaN and ZnO: Theory, measurement, growth, and devices

Applied Physics Reviews 3, 041303 (2016); 10.1063/1.4963919

Accurate determination of optical bandgap and lattice parameters of $\mathrm{Zn}_{1-\mathrm{x}} \mathrm{Mg}_{\mathrm{x}} \mathrm{O}$ epitaxial films $(0 \leq x \leq 0.3)$ grown by plasma-assisted molecular beam epitaxy on a-plane sapphire Journal of Applied Physics 113, 233512 (2013); 10.1063/1.4811693

Patterned silicon substrates: A common platform for room temperature $\mathrm{GaN}$ and $\mathrm{ZnO}$ polariton lasers Applied Physics Letters 104, 241113 (2014); 10.1063/1.4884120

High reflectance dielectric distributed Bragg reflectors for near ultra-violet planar microcavities: $\mathrm{SiO}_{2} / \mathrm{HfO}_{2}$ versus $\mathrm{SiO}_{2} / \mathrm{SiN}_{\mathrm{X}}$

Journal of Applied Physics 120, 093107 (2016); 10.1063/1.4961658

A comprehensive review of $\mathrm{ZnO}$ materials and devices

Journal of Applied Physics 98, 041301 (2005); 10.1063/1.1992666

Polariton lasing in a hybrid bulk $\mathrm{ZnO}$ microcavity

Applied Physics Letters 99, 161104 (2011); 10.1063/1.3650268

\section{Conference Proceedings}




\title{
Homoepitaxial nonpolar (10-10) ZnO/ZnMgO monolithic microcavities: Towards reduced photonic disorder
}

\author{
J. Zuniga-Perez, ${ }^{1, a)}$ L. Kappei, ${ }^{1}$ C. Deparis, ${ }^{1}$ F. Reveret,,${ }^{2,3}$ M. Grundmann, ${ }^{1,4}$ \\ E. de Prado, ${ }^{1,5}$ O. Jamadi, ${ }^{2,3}$ J. Leymarie, ${ }^{2,3}$ S. Chenot, ${ }^{1}$ and M. Leroux ${ }^{1}$ \\ ${ }^{1}$ CRHEA-CNRS, Rue Bernard Gregory, 06560 Valbonne, France \\ ${ }^{2}$ Clermont Université, Institut Pascal (IP), BP 10448, F-63000 Clermont-Ferrand, France \\ ${ }^{3}$ CNRS, UMR 6602, IP, F-63171 Aubière, France \\ ${ }^{4}$ Institut für Experimentelle Physik II, Fakultät für Physik und Geowissenschaften, Universität Leipzig, \\ Linnestr. 5, 04103 Leipzig, Germany \\ ${ }^{5}$ Departamento de Física Aplicada y Electromagnetismo, Universitat de Valencia, c/Dr Moliner 50, \\ Burjassot, Valencia 46100, Spain
}

(Received 2 May 2016; accepted 13 June 2016; published online 24 June 2016)

\begin{abstract}
Nonpolar $\mathrm{ZnO} / \mathrm{ZnMgO}$-based optical microcavities have been grown on (10-10) $\mathrm{m}$-plane $\mathrm{ZnO}$ substrates by plasma-assisted molecular beam epitaxy. Reflectivity measurements indicate an exponential increase of the cavity quality factor with the number of layers in the distributed Bragg reflectors. Most importantly, microreflectivity spectra recorded with a spot size in the order of $2 \mu \mathrm{m}$ show a negligible photonic disorder (well below $1 \mathrm{meV}$ ), leading to local quality factors equivalent to those obtained by macroreflectivity. The anisotropic character of the nonpolar heterostructures manifests itself both in the surface features, elongated parallel to the in-plane $c$ direction, and in the optical spectra, with two cavity modes being observed at different energies for orthogonal polarizations. Published by AIP Publishing. [http://dx.doi.org/10.1063/1.4954796]
\end{abstract}

Semiconductor-based optical microcavities operating at a wavelength $\lambda$ are most often fabricated by sandwiching an active region between two distributed Bragg reflectors (DBRs). DBRs are formed by stacking $\lambda / 4$ bilayers displaying a certain refractive index contrast that imposes, in practice, the number of layers to be stacked to attain a certain reflectivity level. This type of heterostructure forms the backbone of numerous devices, including vertical-cavity surface emitting lasers (VCSEL), ${ }^{1,2}$ and has become of paramount importance in the field of polaritonics. Indeed, the strong-coupling between excitons and cavity photons gives rise to the formation of new eigenstates, the so-called microcavity polaritons, which under certain conditions can undergo a phase transition into a macroscopically-occupied state that emits coherent light, ${ }^{3,4}$ much in the same way as a VCSEL does but with smaller lasing thresholds. Due to the parallelism between the two devices, the former one is now referred to as a polariton laser. ${ }^{5,6}$ For a polariton laser to operate at room-temperature, ${ }^{7,8}$ excitons with high-oscillator strengths and binding energies are beneficial: on the one hand they lead to Rabi splittings sufficiently large for the strong-coupling to be kept up to roomtemperature, and on the other hand they assure stable excitons under large particle densities, necessary to attain the laser threshold. This is the reason why inorganic wide-bandgap semiconductors, including $\mathrm{GaN},{ }^{7} \mathrm{ZnO},{ }^{8}$ and $\mathrm{ZnSe},{ }^{9}$ have been paid so much attention in the last years.

Compared with the most mature GaAs-based microcavities, ${ }^{10-13}$ whose optical quality has been continuously improved, ${ }^{14-17}$ wide-bandgap microcavities still exhibit a large degree of disorder: this disorder is most often associated to the polariton photonic component due to in-plane fluctuations of the cavity or DBRs thicknesses; ${ }^{18,19}$ still, polariton disorder can also display an excitonic component, ${ }^{20}$ particularly if quantum

${ }^{a)}$ Electronic mail: jzp@crhea.cnrs.fr wells (QWs) are used as active medium. The polariton disorder can largely influence the polariton condensation/lasing process, ${ }^{21,22}$ irrespective of its actual origin. In extreme cases, where the disorder is comparable with the system Rabi splitting, it can even lead to the loss of the strong coupling. ${ }^{20} \mathrm{In}$ this respect, current inorganic wide-bandgap microcavities resemble more the first polariton lasers based on $\mathrm{CdTe}^{6,23}$ rather than the current GaAs-based microcavities. ${ }^{10-13}$

In wurtzite materials as $\mathrm{GaN}$ and $\mathrm{ZnO}$ the quantum confined Stark effect ${ }^{24,25}$ (QCSE) exacerbates the excitonic disorder due to quantum wells thickness fluctuations in polar quantum wells. Besides, the spatial separation of the electron and hole wavefunctions due to the QCSE leads to a reduction of the exciton oscillator strength, thereby reducing the achievable Rabi splitting, and renders excitons more sensitive to non-radiative defects due to longer radiative lifetimes. Thus, the growth of nonpolar microcavities $^{26-30}$ is desirable in view of incorporating quantum wells as active regions in $\mathrm{ZnO}$-based microcavities, all of which have been grown up to now along the polar $c$ direction. 19,31-33

In this letter we discuss the fabrication of nonpolar $\mathrm{ZnO} /$ $\mathrm{ZnMgO}$-based microcavities designed to be in the weakcoupling regime (working $\lambda$ of $410 \mathrm{~nm}$, i.e., $\sim 40 \mathrm{~nm}$ below the exciton resonance) and we focus on their linear optical properties, paying special attention to their in-plane homogeneity. Indeed, this will determine the disorder associated to the polariton photonic component.

The heterostructures were grown on $10 \times 20 \mathrm{~mm}^{2}$ nonpolar (10-10) $\mathrm{ZnO}$ substrates, purchased from CRYSTEC, in a Riber Epineat MBE system equipped with effusion cells for elemental $\mathrm{Zn}$ and $\mathrm{Mg}$, and a radio-frequency plasma cell for atomic oxygen (a radiofrequency power of $420 \mathrm{~W}$ was used). The multilayer structures, whatever their nature (i.e., pure $\mathrm{ZnMgO}$ layers, $\mathrm{ZnO} / \mathrm{ZnMgO}$ Bragg reflectors, or complete $\mathrm{ZnO} / \mathrm{ZnMgO}$ microcavities), were grown at a growth temperature of around $400{ }^{\circ} \mathrm{C}$ under $\mathrm{Zn}$-rich conditions, as 
established by monitoring the growth rate as a function of the oxygen flow through the plasma cell. Before introducing the $\mathrm{ZnO}$ substrates into the reactor, substrates were annealed at high temperature $\left(\sim 1100^{\circ} \mathrm{C}\right)$ in an oxygen atmosphere, leading to a step-and-terrace surface as shown in Figure 1(a). The typical root-mean square (RMS) roughness of all the employed substrates was in the order of $0.2 \mathrm{~nm}$ for a 5 $\times 5 \mu \mathrm{m}^{2}$ area, corresponding to the reference value $(0 \times \lambda / 4$ layers) in Figure 1(e). If under the current growth conditions pure $\mathrm{ZnMgO}$ layers with $\mathrm{Mg}$ compositions around 23\% (see Figure 2(a)) are grown, the RMS roughness increases with the deposited thickness, as indicated by the circles in Figure 1(e). The RMS roughness of (10-10) $\mathrm{ZnMgO}$ attains values three times larger than the initial substrate ones for just $400 \mathrm{~nm}$-thick layers. Interestingly, the augmentation of surface roughness with increasing thickness is less pronounced when $\mathrm{ZnO}$ layers are introduced in the heterostructure, represented by the squares in Figure 1(e), which correspond to $\mathrm{ZnO}(\lambda / 4$-thick $) / \mathrm{ZnMgO}(\lambda / 4$-thick $)$ multilayers. While the surface of these layers displays a stripe-like morphology, with elongated features running parallel to the $c$ axis (Figures 1(c) and 1(d)), the surface roughness remains around $1 \mathrm{~nm}$ for more than $3 \mu \mathrm{m}$ thick multilayers, with typical peak-to-valley heights between 3 and $7 \AA$. Furthermore, for a given multilayer structure comprising $66 \times \lambda / 4$ layers, an adjustment of the growth conditions, in particular the $\mathrm{Zn} / \mathrm{O}$ ratio, enables to improve the surface roughness and bring it below $1 \mathrm{~nm}$ (triangle). These roughness values are comparable with those obtained in homoepitaxial layers grown by MBE on more standard polar $\mathrm{ZnO}$ substrates. ${ }^{34}$ They confirm the surface quality of the current multilayers. In the context of polariton condensation and propagation, it is important that the measured surface flatness remains constant at larger scales, typically tens of micrometers, to prevent unintentional condensate localization. This is even more critical on nonpolar microcavities, where step bunching might create large thickness fluctuations decreasing the local quality factor $(\mathrm{Q}){ }^{35}$ As clearly displayed in Figure 1(f), the RMS roughness is independent of the surface area and can be kept below $1 \mathrm{~nm}$ even for the $66 \times \lambda / 4$ layers structure.

If large- $Q$ and homogeneous cavities are to be obtained, the previous in-plane uniformity must be accompanied by constant $\mathrm{Mg}$ composition in consecutive $\mathrm{ZnMgO}$ layers. Secondary ion mass spectrometry (SIMS) profiles of nonpolar $\mathrm{ZnMgO}$ layers display a constant $\mathrm{Mg}$ concentration (Figure 2(a)), even if the layer thickness is larger than the critical thickness for plastic relaxation. ${ }^{36}$ Indeed, it should be kept in mind for the rest of the article that not only the $400 \mathrm{~nm}$ thick $\mathrm{ZnMgO}$ layer is cracked, but also all the heterostructures displaying more than $20 \times \lambda / 4$ layers. ${ }^{36}$ The observed homogenity of $\mathrm{Mg}$ composition along the growth direction is consistent with previous studies on heteroepitaxial nonpolar MBE-grown $\mathrm{ZnMgO}$ layers. ${ }^{37}$ However, we have observed that for long growth runs, typically longer than $10 \mathrm{~h}$, a slight increase of $\mathrm{Mg}$ composition within consecutive $\mathrm{ZnMgO}$ layers occurs, as observed in Figure 2(b), concomitantly with a slight decrease in the thickness of the individual $\mathrm{ZnO}$ layers. These slow variations, compared with the growth time of individual $\lambda / 4$ layers, should be taken into

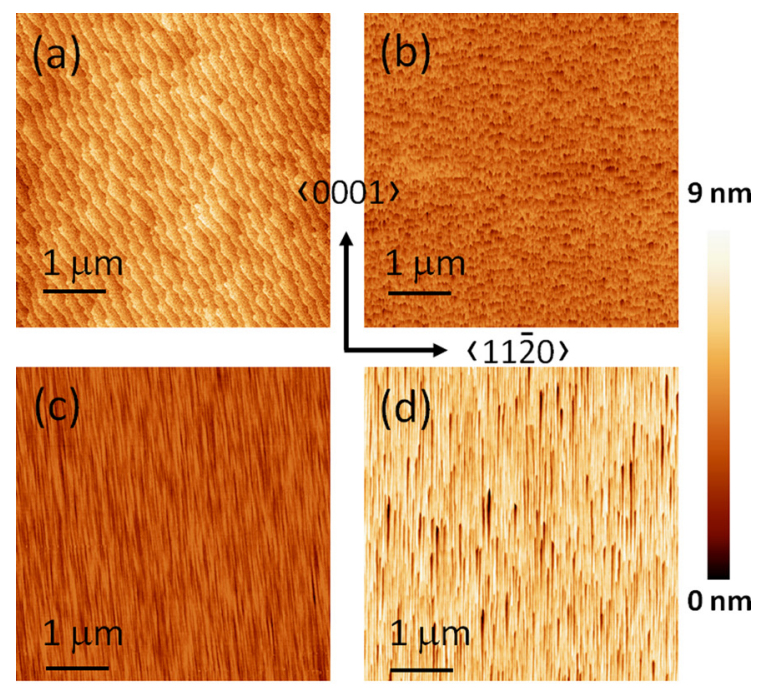

Thickness (nm)
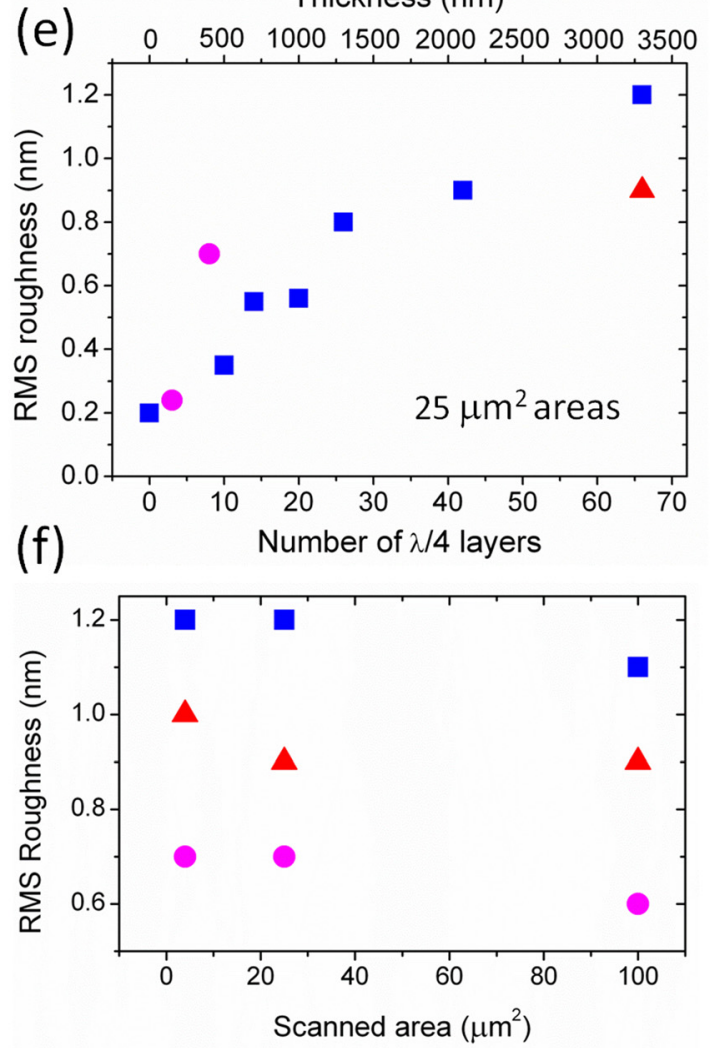

FIG. 1. Atomic force microscopy images of: (a) an annealed (10-10) $\mathrm{ZnO}$ substrate, (b) a 400 nm-thick $\mathrm{Zn}_{0.77} \mathrm{Mg}_{0.23} \mathrm{O}$ layer, (c), a $10 \times(\mathrm{ZnO} / \mathrm{ZnMgO}) \mathrm{DBR}$, and $(\mathrm{d})$ a $16 \times(\mathrm{ZnO} / \mathrm{ZnMgO}) \mathrm{DBR} / \lambda(\mathrm{ZnO}) / 15 \times(\mathrm{ZnO} / \mathrm{ZnMgO}) \mathrm{DBR}$ microcavity. The color scale applies to panels (b)-(d). The orientation of the crystallographic directions applies to all four AFM images with an uncertainty ( $\sim$ several degrees) due to the exact sample orientation in the AFM. (e) RMS roughness plotted as a function of the number of $\lambda / 4$ layers (or equivalent thickness for pure $\mathrm{ZnMgO}$ layers) for pure $\mathrm{ZnMgO}$ layers (circles), $\mathrm{ZnO} / \mathrm{ZnMgO}$ multilayers (DBRs and full microcavities) grown under constant growth conditions (squares), and under further optimized $\mathrm{Zn} / \mathrm{O}$ ratio (triangle). (f) RMS roughness as a function of the size of the scanned area for the $66 \times \lambda / 4$ thick heterostructures in (e) and for the $8 \times \lambda / 4$ thick $\mathrm{ZnMgO}$ layer in (e).

account and corrected for in thick microcavity structures as the one illustrated in Figure 2(c), which displays the cross section of a $16 \times(\mathrm{ZnO} / \mathrm{ZnMgO}) \mathrm{DBR} / \lambda(\mathrm{ZnO}) / 15 \times(\mathrm{ZnO} /$ $\mathrm{ZnMgO}) \mathrm{DBR}$ microcavity structure.

While most $\mathrm{ZnO}$ microcavities are based either on nitride- ${ }^{19,21,33}$ or on dielectric-DBRs, ${ }^{31,38}$ it was shown in the 

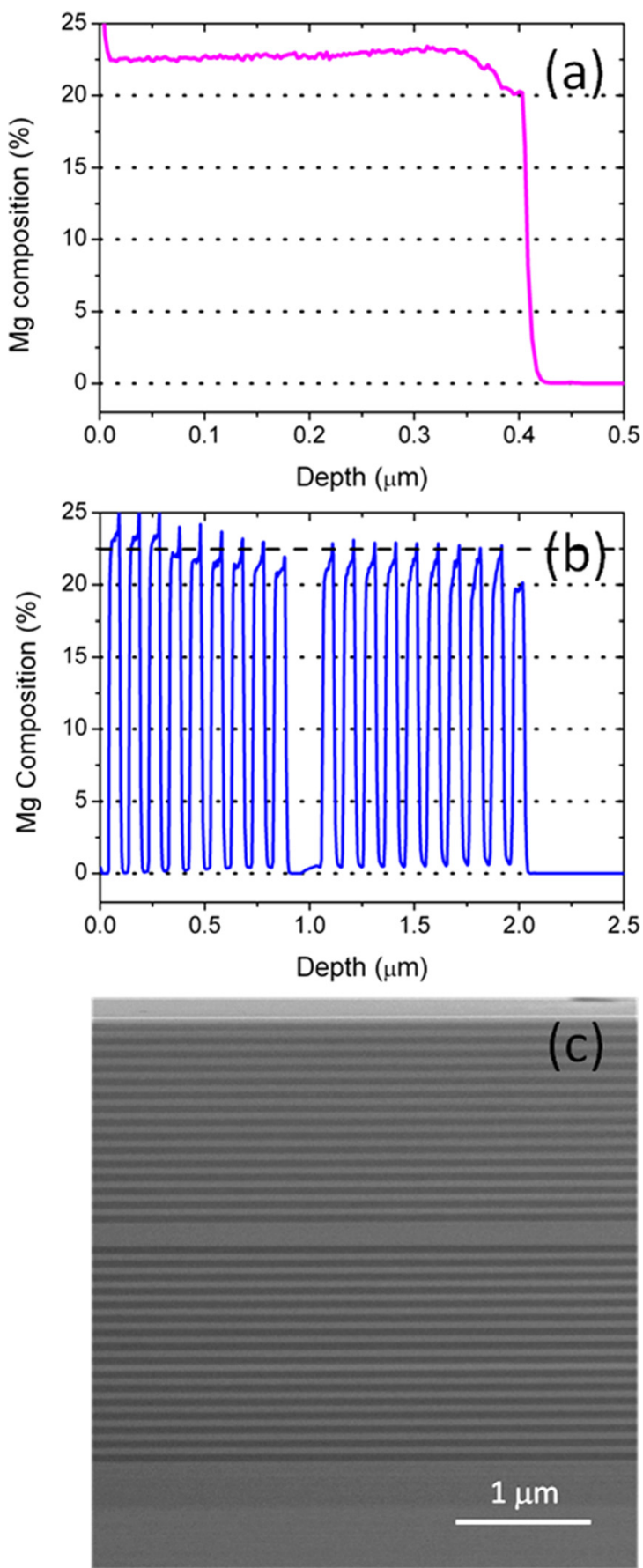

FIG. 2. SIMS profile of the $\mathrm{Mg}$ concentration in (a) a pure $\mathrm{ZnMgO}$ layer $(400 \mathrm{~nm}$ thick) and (b) $10 \times(\mathrm{ZnO} / \mathrm{ZnMgO}) \mathrm{DBR} / \lambda(\mathrm{ZnO}) / 9 \times(\mathrm{ZnO} /$ $\mathrm{ZnMgO}$ )DBR microcavity. The dashed line in (b) is just a guide for the eyes. (c) Cross-section scanning electron microscopy image of a $16 \times(\mathrm{ZnO} /$ $\mathrm{ZnMgO}) \mathrm{DBR} / \lambda(\mathrm{ZnO}) / 15 \times(\mathrm{ZnO} / \mathrm{ZnMgO}) \mathrm{DBR}$ microcavity.

$2010 \mathrm{~s}$ that monolithic ZnO-based DBRs and microcavities were feasible by combining either $\mathrm{ZnO}$ and $\mathrm{ZnMgO}$ layers, or layers of $\mathrm{ZnMgO}$ with different $\mathrm{Mg}$ compositions. ${ }^{32}$ Indeed, VCSEL operation in polar ZnO-based microcavities was demonstrated almost simultaneously in a monolithic and in a nitride-DBR cavity, ${ }^{32,39}$ illustrating the possibilities of monolithic cavities. Compared with the first monolithic DBRs, which were grown along the polar direction, ${ }^{32,40}$ nonpolar DBRs display a polarization-dependent stopband position, as seen for a $10 \times(\mathrm{ZnO} / \mathrm{ZnMgO}) \mathrm{DBR}$ in Figure 3(a). This shift arises due to the $\mathrm{ZnO}$ and $\mathrm{ZnMgO}$ birefringence, ${ }^{41}$ and has been also observed in nonpolar nitride DBRs. ${ }^{26-28}$ For the same reason, two orthogonally-polarized cavity modes are detected at normal incidence in full microcavity structures containing a central $\mathrm{ZnO} \lambda$ layer (designed for a wavelength of about $410 \mathrm{~nm}$, i.e., $3.02 \mathrm{eV}$ ), as shown in Figure 3(b). The solid and dashed spectra correspond to polarizations parallel and perpendicular to the in-plane wurtzite $c$-direction, respectively. The fact that for these wavelengths the cavity mode polarized perpendicular to the $c$-axis is observed at higher energies (i.e., lower wavelengths) than the parallel one is consistent with the isotropic point in $\mathrm{ZnO}$ being found between $395 \mathrm{~nm}(3.14 \mathrm{eV})$ and $400 \mathrm{~nm}$ $(3.10 \mathrm{eV})$, combined with a positive $\mathrm{ZnO}$ birefringence
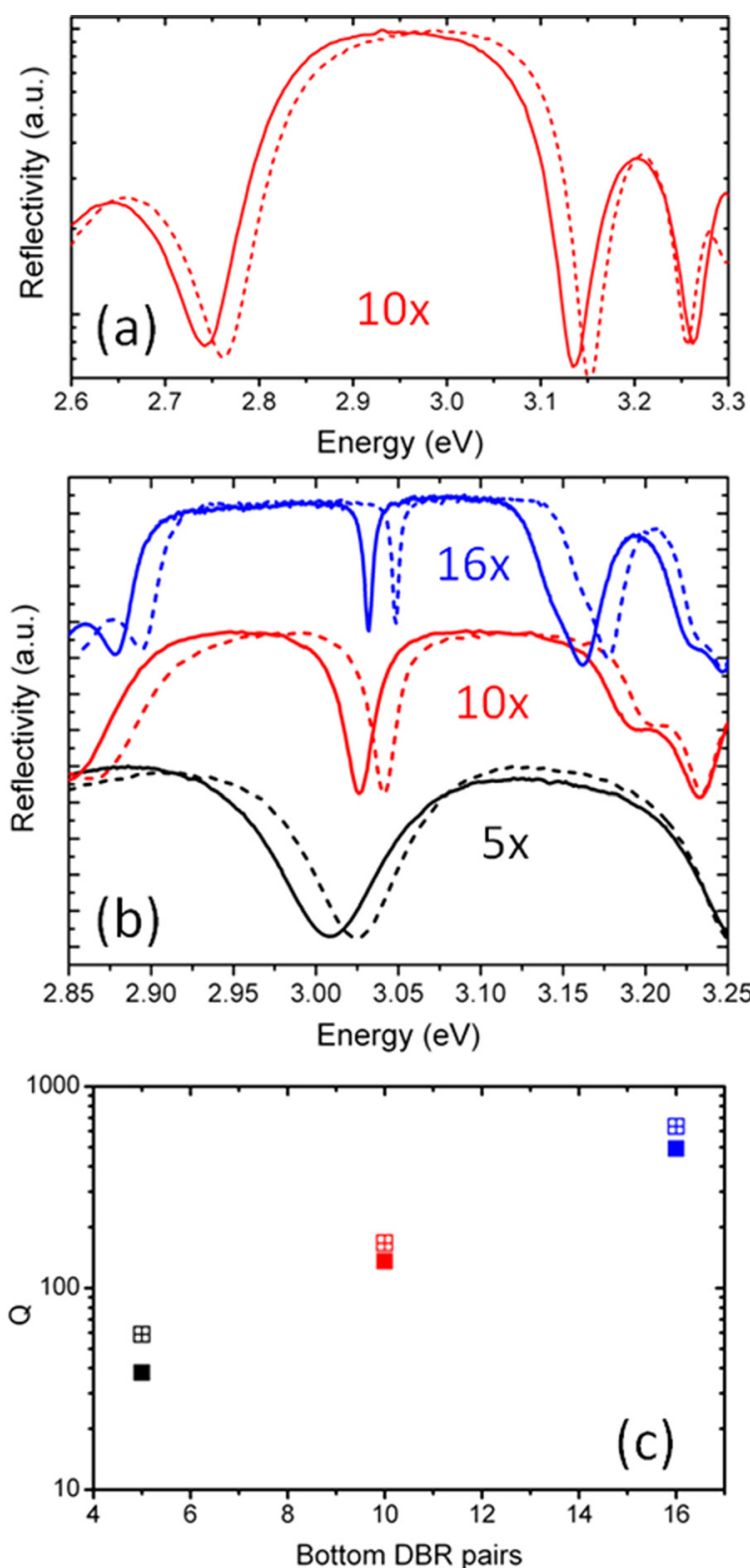

FIG. 3. Polarization-resolved room-temperature macroreflectivity measurements at normal incidence on: (a) $10 \times(\mathrm{ZnO} / \mathrm{ZnMgO}) \mathrm{DBR}$, and (b) $\mathrm{m}$ $\times(\mathrm{ZnO} / \mathrm{ZnMgO}) \mathrm{DBR} / \lambda(\mathrm{ZnO}) / \mathrm{n} \times(\mathrm{ZnO} / \mathrm{ZnMgO}) \mathrm{DBR}$ microcavities with $\mathrm{n}=\mathrm{m}-1$ and $\mathrm{m}=5,10$, and 16 . The solid and dashed lines correspond to polarizations parallel and perpendicular to the $c$-axis, respectively. In (b) the spectra are offset vertically for clarity. (c) Q as a function of the number of pairs in the bottom DBR - the top one having one pair less-for the cavity modes shown in (b). Full and dashed squares correspond to polarizations parallel and perpendicular to the $c$-axis, respectively. 
$\left(\mathrm{n}_{\text {par }}-\mathrm{n}_{\text {perp }}\right)$ for wavelengths larger than the isotropic point. ${ }^{42,43}$ Furthermore, if we assume that the cavity mode is completely confined in the central $\mathrm{ZnO} \lambda$-thick layer, then we can estimate the $\mathrm{ZnO}$ birefringence to be close to 0.01 , as determined experimentally in $\mathrm{ZnO}$ single crystals. ${ }^{42,43}$

As stated in the introduction, in order to enhance the reflectivity of the DBRs and enhance the photonic confinement within a microcavity, the number of bilayers constituting the DBRs must be increased. This is illustrated in Figure 3(c), where the experimental Qs for each polarization (extracted by fitting the cavity modes with pure Lorentzians) are plotted as a function of the number of pairs in the bottom DBR, the top one containing one pair less. While these Qs are smaller than the state-of-the-art Qs in polar $\mathrm{ZnO}$ microcavities, obtained with nitride- and dielectric-DBRs and currently attaining several thousands, ${ }^{19,38}$ they are indeed of the same order of magnitude as those obtained previously with a polar monolithic approach. ${ }^{32}$ Furthermore, it should be noted that the Qs corresponding to a polarization perpendicular to the $c$-axis are larger than those of the polarization parallel to the $c$ direction; this observation is consistent with the birefringence evolution as a function of $\mathrm{Mg}$ composition (i.e., larger birefringence than $\mathrm{ZnO}$ and same sign) measured in Ref. 44.

Most importantly, the Qs determined by macroscopic reflectivity measurements (spot diameter in the order of $100 \mu \mathrm{m})$ are equivalent to those obtained with a spot of about $2 \mu \mathrm{m}$, as evidenced in Figure 4(a). This is a first indication of a large spatial homogeneity and of a reduced photonic disorder. ${ }^{18,19}$ It should be noted that we have performed the microreflectivity measurements in Fourier imaging configuration, with an objective NA of 0.4 , and that the signal normal to the surface has been filtered out from the overall signal, which covers otherwise a light cone of $\pm 23^{\circ}$. To further confirm this, polarization-resolved microreflectivity spectra have been acquired every $10 \mu \mathrm{m}$ close to the sample border (about $1 \mathrm{~mm}$ from the sample limit), where the inhomogeneities are expected to be larger. Four of such spectra (separated by $50 \mu \mathrm{m}$ ) are shown in Figure 4(b) for one polarization. The analysis of the microreflectivity spectra acquired along a line $150 \mu \mathrm{m}$ long indicate a small shift of the cavity modes, corresponding to photonic gradients of $20( \pm 4) \mu \mathrm{eV} /$ $100 \mu \mathrm{m}$ for the polarization perpendicular to the $c$ direction, and of $57( \pm 8) \mu \mathrm{eV} / 100 \mu \mathrm{m}$ for the polarization parallel to the $c$ direction (see linear fits in Figure 4(c)). These photonic gradients are of the same order of magnitude as the one suggested by Figure 4(a). In it the macroreflectivity spectra were acquired at the sample center and are redshifted $8-9 \mathrm{meV}$ of the microreflectivity spectra acquired at the sample border, about $10 \mathrm{~mm}$ away. If we consider a homogeneous energy gradient, these numbers result in a photonic gradient of about $80-90 \mu \mathrm{eV} / 100 \mu \mathrm{m}$, compatible with those extracted from Figure 4(c). Similarly, the extracted full width at half maxima of the cavity modes display fluctuations in the order of hundreds of $\mu \mathrm{eV}$. These two figures of merit are at least one order of magnitude better than those of previous reports, and show the full potential of $\mathrm{ZnO}$-based nonpolar monolithic microcavities for studying polariton condensates propagation within a very homogenous photonic potential landscape. The reasons for the current improvement, compared with other wide bandgap microcavities where the photonic homogeneity
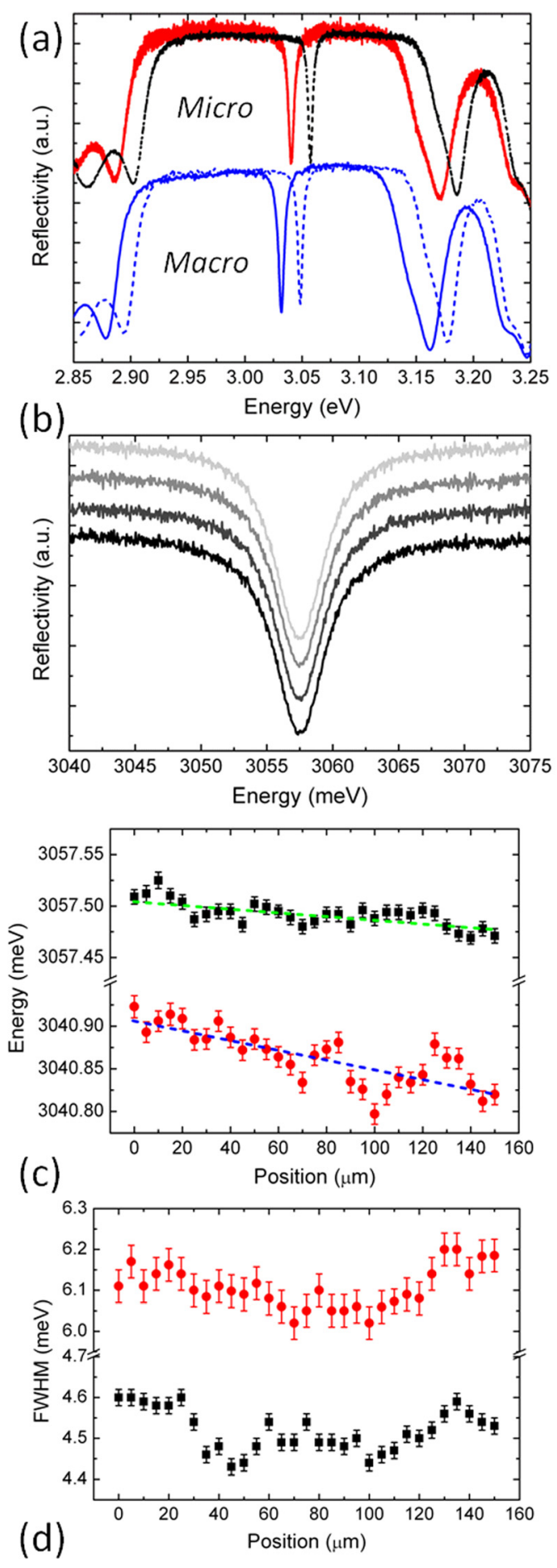

FIG. 4. (a) Polarization-resolved room-temperature macroreflectivity and microreflectivity measurements at normal incidence on a $16 \times(\mathrm{ZnO} /$ $\mathrm{ZnMgO}) \mathrm{DBR} / \lambda(\mathrm{ZnO}) / 15 \times(\mathrm{ZnO} / \mathrm{ZnMgO}) \mathrm{DBR}$ microcavity. The macroreflectivity (diameter about $100 \mu \mathrm{m}$ ) spectra were acquired on the sample center whereas the microreflectivity (diameter about $2 \mu \mathrm{m}$ ) ones were measured close to the sample border. The solid and dashed lines correspond to polarizations parallel and perpendicular to the $c$-axis, respectively. The microreflectivity spectra were offset vertically for clarity. (b) Microreflectivity spectra acquired every $50 \mu \mathrm{m}$ close to a sample border for a polarization perpendicular to the $c$-axis. (c) Energy of the cavity modes extracted from polarization-resolved microreflectivity measurements performed every $10 \mu \mathrm{m}$ close to the sample border: polarization perpendicular (squares) and parallel (circles) to the $c$-axis. The dashed lines correspond to linear fits of the data. (d) FWHM extracted from polarization-resolved microreflectivity measurements performed every $10 \mu \mathrm{m}$ close to the sample border (same spectra as in (c)): polarization perpendicular (squares) and parallel (circles) to the $c$-axis. All measurements were carried out at room-temperature and the cavity modes were fitted with pure Lorentzians. 
has been investigated, ${ }^{18,19,21,45}$ can be the low growth temperature, which helps in preventing any interdiffusion between $\mathrm{ZnO}$ and $\mathrm{ZnMgO}$ thereby allowing for abrupt interfaces, and the insensitivity of the $\mathrm{ZnO} / \mathrm{ZnMgO}$ surface features and roughness to the initial substrate miscut, which renders less critical the exact unintentional substrate miscut. ${ }^{46}$

To conclude, we have introduced the growth of homoepitaxial nonpolar (10-10) monolithic $\mathrm{ZnO} / \mathrm{ZnMgO}$ optical microcavities displaying flat surfaces and homogeneous $\mathrm{Mg}$ composition, even for micrometer-thick heterostructures. The possibility of stacking a large number of $\lambda / 4$ bilayers has enabled us to characterize optical microcavities with Qs in the order of 600 and displaying a photonic disorder one order of magnitude smaller than the state-of-the-art in wide bandgap microcavities, reducing the gap with the most developed GaAs-based microcavities. Still, an open issue remains concerning the exploitation of such improved photonic figures of merit in strongly-coupled microcavities, especially in terms of the active region to be used. Several open possibilities exist, including the use of $\mathrm{ZnCdO}$ QWs or pure $\mathrm{ZnCdO}$ layers with low $\mathrm{Cd}$ content, to limit the associated inhomogeneous broadening, ${ }^{20}$ as well as the use of $\mathrm{ZnMgO} / \mathrm{ZnMgO}$-based DBRs and $\mathrm{ZnO}$ as active region, ${ }^{32}$ the problem being in this last situation the reduction of the DBR stopband. ${ }^{19}$ One might think to combine the advantages of both solutions and try to mitigate, thereby, their respective disadvantages.

The authors would like to acknowledge F. Semond, P. Vennéguès, and M. Richard for fruitful discussions and V. Brändli and O. Tottereau for helpful support in the SEM and EDX measurements. E. de Prado thanks Ministerio de Economia y Competitividad for financial support under Grant No. EEBB-I-15-09749.

${ }^{1}$ T. Someya, R. Werner, A. Forchel, M. Catalano, R. Cingolani, and Y. Arakawa, Science 285, 1905 (1999).

${ }^{2}$ D. Kasahara, D. Morita, T. Kosugi, K. Nakagawa, J. Kawamata, Y. Higuchi, H. Matsumura, and T. Mukai, Appl. Phys. Express 4, 072103 (2011).

${ }^{3}$ A. V. Kavokin, J. J. Baumberg, G. Malpuech, and F. P. Laussy, Microcavities (Oxford University Press, New York, 2007).

${ }^{4}$ J. Kasprzak, M. Richard, S. Kundermann, A. Baas, P. Jeambrun, J. M. J. Keeling, F. Marchetti, M. H. Szymanska, R. André, J. L. Staehli, V. Savona, P. B. Littlewood, B. Deveaud, and Le. Si Dang, Nature 443, 409 (2006).

${ }^{5}$ A. Imamoglu, R. J. Ram, S. Pau, and Y. Yamamoto, Phys. Rev. A 53, 4250 (1996).

${ }^{6}$ J. Kazprzak, D. D. Solnyshkov, R. André, Le. Si Dang, and G. Malpuech, Phys. Rev. Lett. 101, 146404 (2008).

${ }^{7}$ S. Christopoulos, G. B. Höger von Högersthal, J. J. Baumberg, A. J. D. Grundy, P. G. Lagoudakis, A. V. Kavokin, G. Christmann, R. Butté, E. Feltin, J. F. Carlin, and N. Grandjean, Phys. Rev. Lett. 98, 126405 (2007). ${ }^{8} \mathrm{~F}$. Li, L. Orosz, O. Kamoun, S. Bouchoule, C. Brimont, P. Disseix, T. Guillet, X. Lafosse, M. Leroux, J. Leymarie, M. Mexis, M. Mihailovic, G. Patriarche, F. Réveret, D. Solnyshkov, J. Zuniga-Perez, and G. Malpuech, Phys. Rev. Lett. 110, 196406 (2013).

${ }^{9}$ T. Klein, S. Klembt, E. Durupt, C. Hruse, D. Hommel, and M. Richard, Appl. Phys. Lett. 107, 071101 (2015).

${ }^{10}$ B. Nelsen, G. Liu, M. Steger, D. W. Snoke, R. Balili, K. West, and L. Pfeiffer, Phys. Rev. X 3, 041015 (2013).

${ }^{11}$ C. Sturm, D. Tanese, H. S. Nguyen, H. Flayac, E. Galopin, A. Lemaitre, I. Sagnes, D. Solnyshkov, A. Amo, G. Malpuech, and J. Bloch, Nat. Commun. 5, 3278 (2014)

${ }^{12}$ P. Cilibrizzi, A. Askitopoulos, M. Silva, F. Bastiman, E. Clarke, J. M. Zajac, W. Langbein, and P. G. Lagoudakis, Appl. Phys. Lett. 105, 191118 (2014).

${ }^{13}$ L. Tinkler, P. M. Walker, E. Clarke, D. N. Krizhanovskii, F. Bastiman, M. Durska, and M. S. Skolnick, Appl. Phys. Lett. 106, 021109 (2015).

${ }^{14}$ M. Gurioli, F. Bogani, D. S. Wiersma, P. Roussignol, G. Cassabois, G. Khitrova, and H. Gibbs, Phys. Rev. B 64, 165309 (2001).
${ }^{15}$ W. Langbein and J. M. Hvam, Phys. Rev. Lett. 88, 047401 (2002).

${ }^{16}$ M. Abbarchi, C. Diederichs, L. Largeau, V. Ardizzone, O. Mauguin, T. Lecomte, A. Lemaitre, J. Bloch, P. Roussignol, and J. Tignon, Phys. Rev. B 85, 045316 (2012).

${ }^{17}$ J. M. Zajac, W. Langbein, M. Hugues, and M. Hopkinson, Phys. Rev. B 85, 165309 (2012).

${ }^{18}$ G. Christmann, R. Butté, E. Feltin, J. F. Carlin, and N. Grandjean, Appl. Phys. Lett. 89, 261101 (2006).

${ }^{19}$ J. Zuniga-Perez, E. Mallet, R. Hahe, M. J. Rashid, S. Bouchoule, C. Brimont, P. Disseix, J. Y. Duboz, G. Gommé, T. Guillet, O. Jamadi, X. Lafosse, M. Leroux, J. Leymarie, Feng Li, F. Réveret, and F. Semond, Appl. Phys. Lett. 104, 241113 (2014).

${ }^{20}$ M. Glauser, C. Mounir, G. Rossbach, E. Feltin, J. F. Carlin, R. Butté, and N. Grandjean, J. Appl. Phys. 115, 233511 (2014).

${ }^{21}$ O. Jamadi, F. Réveret, E. Mallet, P. Disseix, F. Médard, M. Mihailovic, D. Solnyshkov, G. Malpuech, J. Leymarie, X. Lafosse, S. Bouchoule, F. Li, M. Leroux, F. Semond, and J. Zuniga-Perez, Phys. Rev. B 93, 115205 (2016).

${ }^{22}$ M. Thunert, A. Janot, H. Franke, C. Sturm, T. Michalsky, M. D. Martin, L. Viña, B. Rosenow, M. Grundmann, and R. Schmidt-Grund, Phys. Rev. B 93, 064203 (2016).

${ }^{23}$ M. Richard, J. Kasprzak, R. André, R. Romestain, Le. Si Dang, G. Malpuech, and A. Kavokin, Phys. Rev. B 72, 201301 (2005).

${ }^{24}$ M. Leroux, N. Grandjean, M. Laügt, J. Massies, B. Gil, P. Lefebvre, and P. Bigenwald, Phys. Rev. B 58, 13371 (1998).

${ }^{25}$ C. Morhain, T. Bretagnon, P. Lefebvre, X. Tang, P. Valvin, T. Guillet, B. Gil, T. Taliercio, M. Teisseire-Doninelli, B. Vinter, and C. Deparis, Phys. Rev. B 72, 241305 (2005).

${ }^{26}$ T. Zhu, A. Dussaigne, G. Christmann, C. Pinquier, E. Feltin, D. Martin, R. Butté, and N. Grandjean, Appl. Phys. Lett. 92, 061114 (2008).

${ }^{27}$ G. Rossbach, J. Levrat, A. Dussaigne, G. Cosendey, M. Glauser, M. Cobet, R. Butté, N. Grandjean, H. Teisseyre, M. Bockowski, I. Grzegory, and T. Suski, Phys. Rev. B 84, 115315 (2011).

${ }^{28}$ J. Levrat, G. Rossbach, A. Dussaigne, G. Cosendey, M. Glauser, M. Cobet, R. Butté, N. Grandjean, H. Teisseyre, M. Bockowski, I. Grzegory, and T. Suski, Phys. Rev. B 86, 165321 (2012).

${ }^{29}$ R. Tao, M. Arita, S. Kako, and Y. Arakawa, Phys. Stat. Sol. C 11, 840 (2014).

${ }^{30}$ R. Tao, M. Arita, S. Kako, K. Kamide, and Y. Arakawa, Appl. Phys. Lett. 107, 101102 (2015).

${ }^{31}$ H. Franke, C. Sturm, R. Schmidt-Grund, G. Wagner, and M. Grundmann, New J. Phys. 14, 013037 (2012).

${ }^{32}$ S. Kalusniak, S. Sadofev, S. Halm, and F. Hennerberger, Appl. Phys. Lett. 98, 011101 (2011).

${ }^{33}$ Y. Y. Lai, Y. H. Chou, Y. P. Lan, T. C. Lu, S. C. Wang, and Y. Yamamoto, Sci. Rep. 6, 20581 (2016).

${ }^{34}$ S. Sadofev, P. Schäfer, Y. H. Fan, S. Blumstengel, and F. Hennerberger, Appl. Phys. Lett. 91, 201923 (2007).

${ }^{35}$ R. Tao, M. Arita, S. Kako, and Y. Arakawa, Appl. Phys. Lett. 103, 20118 (2013).

${ }^{36}$ J. Zuniga-Perez, "Strain relaxation in homoepitaxial $\mathrm{ZnMgO} / \mathrm{ZnO}$ heterostructures: nonpolar Vs semipolar," J. Appl. Phys. (unpublished).

${ }^{37}$ B. Laumer, F. Schuster, M. Stutzmann, A. Bergamier, G. Dollinger, and M. Eickhoff, J. Appl. Phys. 113, 233512 (2013).

${ }^{38}$ F. Li, L. Orosz, O. Kamoun, S. Bouchoule, C. Brimont, P. Disseix, T. Guillet, X. Lafosse, M. Leroux, J. Leymarie, G. Malpuech, M. Mexis, M. Mihailovic, G. Patriarche, F. Réveret, D. Solnyshkov, and J. Zuniga-Perez, Appl. Phys. Lett. 102, 191118 (2013).

${ }^{39}$ T. Guillet, C. Brimont, P. Valvin, B. Gil, T. Bretagnon, F. Médard, M. Mihailovic, J. Zuniga-Perez, M. Leroux, F. Semond, and S. Bouchoule, Appl. Phys. Lett. 98, 211105 (2011).

${ }^{40}$ S. Halm, S. Kalusniak, S. Sadofev, H. J. Wünsche, and F. Hennerberger, Appl. Phys. Lett. 99, 181121 (2011).

${ }^{41}$ R. Schmidt, B. Rheinländer, M. Schubert, D. Spemann, T. Butz, J. Lenzner, E. M. Kaidashev, M. Lorenz, A. Rahm, H. C. Semmelhack, and M. Grundmann, Appl. Phys. Lett. 82, 2260 (2003).

${ }^{42}$ Y. S. Park and J. R. Schneider, J. Appl. Phys. 39, 3049 (1968).

${ }^{43}$ G. E. Jellison, Jr. and C. M. Rouleau, Appl. Opt. 44, 3153 (2005).

${ }^{44}$ C. W. Teng, J. F. Muth, U. Ozgur, M. J. Bergmann, H. O. Everitt, A. K. Sharma, C. Jin, and J. Narayan, Appl. Phys. Lett. 76, 979 (2000).

${ }^{45}$ Z. Gacevic, G. Rossbach, R. Butté, F. Réveret, M. Glauser, J. Levrat, G. Cosendey, J. F. Carlin, N. Grandjean, and E. Calleja, J. Appl. Phys. 114, 233102 (2013).

${ }^{46}$ R. M. Farrell, D. A. Haeger, X. Chen, M. Iza, A. Hirai, K. M. Kelchner, K. Fujito, A. Chakraborty, S. Keller, S. P. DenBaars, J. S. Speck, and S. Nakamura, J. Cryst. Growth 313, 1 (2010). 